\section{Note \\ Myoglobin-catalyzed Peroxidation Products of Methyl Eicosapentaenoate}

\author{
Ryo Yamauchi, Tomoo Yamada, Koji Kato \\ and Yoshimitsu UENO
}

Department of Agricultural Chemistry, Gifu University, Gifu 501-11, Japan

Received March 26, 1985

Heme compounds catalyze the decomposition of fatty acid hydroperoxides, and free radical intermediates generated during this process initiate oxygenation of unsaturated fatty acids. ${ }^{1)}$ Since heme compounds frequently occur in the proximity of oxygen and unsaturated lipids in biological systems, heme catalyzed reactions can be initiated readily if peroxides form in this environment. Eicosapentaenoic acid is a constituent of fish oils, and is susceptible to lipid peroxidation because of the presence of five double bonds in the molecule. Therefore, eicosapentaenoic acid may be subjected to heme-catalyzed peroxidation. In our previous papers, ${ }^{2,3)}$ we reported the results of a gas chromatography-mass spectrometric (GCMS) analysis of several oxygenated products formed by the autoxidation and photosensitized oxidation of methyl eicosapentaenoate. This paper deals with the products formed by myoglobin-catalyzed peroxidation of methyl eicosapentaenoate.

Methyl eicosapentaenoate was purified from methyl eicosapentaenoate concentrate, which was provided by Taiyo Fishery Co., $\mathrm{Ltd}^{2}{ }^{2}$ The purity of the methyl eicosapentaenoate was $>99 \%$ by gas liquid chromatography (GLC). Myoglobin Type II, purchased from Sigma Chem. Co., was used without further purification.

Methyl eicosapentaenoate $(300 \mathrm{mg})$ containing myoglobin $(0.3 \mathrm{mg})$ was oxidized for $8 \mathrm{hr}$ at $37^{\circ} \mathrm{C}$ in the dark. The oxidation products were analyzed by thin layer chromatography (TLC) with hexane-ethyl ether-acetic acid $(60: 40: 1, \mathrm{v} / \mathrm{v})$ as the solvent system. $\left.{ }^{2}\right)$ Figure 1 shows the densitometric pattern of the myoglobin-catalyzed peroxidation products of methyl eicosapentaenoate. Spots corresponding to methyl eicosapentaenoate and several oxidation products were observed on the TLC plate. The oxidation products, $\mathbf{A}, \mathbf{B}$ and $\mathbf{C}$, were isolated by silica gel column chromatography, ${ }^{3)}$ and their structures were analyzed by the methods described previously.,3) The structures of $\mathbf{A}, \mathbf{B}$ and $\mathbf{C}$ were found to be the same as the autoxidation products of methyl eicosapentaenoate; ${ }^{2,3}$ ) that is, $\mathbf{A}$, the eight regioisomers of methyl eicosapentaenoate monohydroperoxide; $\mathbf{B}$, the two isomers of hydroperoxy five-membered endoperoxide; and $\mathbf{C}$, the eight isomers of prostaglandin-like hydroperoxy bicyclic en-

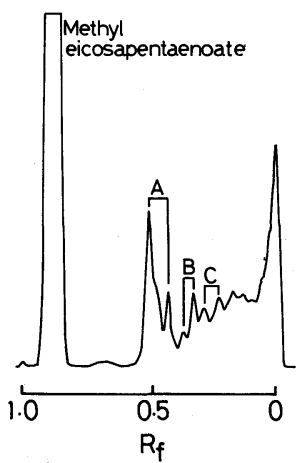

FIG. 1. Densitometric Pattern of Myoglobin-catalyzed Peroxidation Products of Methyl Eicosapentaenoate on the TLC Plate.

An aliquot of the oxidized methyl eicosapentaenoate (for $8 \mathrm{hr}$ at $37^{\circ} \mathrm{C}$ ) was spotted on a TLC plate. The plate was developed with a solvent of hexane-ethyl ether-acetic acid $(60: 40: 1, \mathrm{v} / \mathrm{v})$. After it had been sprayed with saturated $\mathrm{K}_{2} \mathrm{Cr}_{2} \mathrm{O}_{7}$ in $50 \% \mathrm{H}_{2} \mathrm{SO}_{4}$ solution, the plate was heated for $10 \mathrm{~min}$ at $110^{\circ} \mathrm{C}$ and monitored by a densitometer at $380 \mathrm{~nm}$.

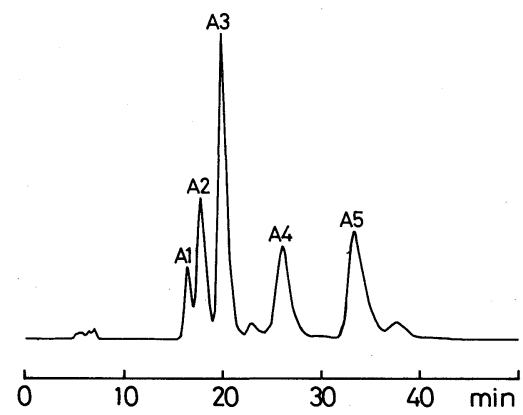

FIG. 2. High Performance Liquid Chromatogram of Methyl Eicosapentaenoate Monohydroperoxides.

Detection was based on the absorbance at $205 \mathrm{~nm}$.

doperoxide. The 5,18-dihydroperoxide which was a product of autoxidized methyl eicosapentaenoate was not detected in this reaction system.

The isomers of methyl eicosapentaenoate monohydroperoxide were separated by high performance liquid chromatography (HPLC) on a column $(4.6 \times 250 \mathrm{~mm})$ of LiChrosorb Si 60 (Merck, $10 \mu \mathrm{m}$ particle size), using $0.5 \%$ $(\mathrm{v} / \mathrm{v})$ 2-propanol in hexane at a flow rate of $1.0 \mathrm{ml} / \mathrm{min}$. The compounds were detected according to their absorbance at $205 \mathrm{~nm}$. Figure 2 shows the HPLC pattern of methyl eicosapentaenoate monohydroperoxides. Five peaks, A1, A2, A3, A4 and A5, appeared on the chromatogram. The five peaks were fractionated, and their structures were analyzed by GC-MS after hydrogenation and trimethylsilylation. ${ }^{2)}$ A1, A2, A4 and A5 gave one peak on each gas chromatogram, and A3 gave two peaks. From the mass spectrum of each peak on the gas chromatogram, 
the monohydroperoxides separated by HPLC were assigned to be regioisomeric monohydroperoxides as shown in Table I. The relative amounts of monohydroperoxide isomers were determined by HPLC. The amounts of the 11- and 18-isomers, which were not separated by HPLC, were determined by GLC after hydrogenation and trimethylsilylation. ${ }^{2)}$ Table II shows the isomeric distributions of methyl eicosapentaenoate monohydroperoxides formed by myoglobin-catalyzed peroxidation. The distributions of monohydroperoxide isomers were the same as those obtained for autoxidation, and did not change during the peroxidation process. On the other hand, the regioisomers of monohydroperoxide did not equally distribute. The outer isomers (the hydroperoxy group located outside the pentaene structure, the 5- and 18-isomers) had higher yields than the inner isomers (the hydroperoxy group located inside the pentaene structure, the 8-, 9-, 11-, 12-, 14- and 15-isomers). The same tendencies have been reported with the compositions of methyl linolenate monohydroperoxide isomers, ${ }^{4.5)}$ arachidonic acid monohydroperoxide isomers ${ }^{6)}$ and methyl arachidonate monohydroperoxide isomers. ${ }^{6 \sim 8)}$ Porter et al. ${ }^{9)}$ has suggested that the peroxy radicals which have double bonds at
TABle I. GC-MS ANAlysis OF ISOMERIC METhyL MONOHYDROXY EICOSANOATE TRIMETHYLSILYL Derivatives from Each Peak of Methyl EicosapentaenOATE MONOHYDROPEROXIDES ON HPLC

\begin{tabular}{cccc}
\hline \multirow{2}{*}{ HPLC peak } & \multicolumn{2}{c}{$\begin{array}{c}m / z \text { for } \alpha \text {-cleavage of the } \\
\text { trimethylsilyloxy group }\end{array}$} & \\
\cline { 2 - 3 } & $\begin{array}{c}\text { Toward the } \\
\mathrm{COOCH}_{3} \text { group }\end{array}$ & $\begin{array}{c}\text { Toward the } \\
\mathrm{CH}_{3} \text { group }\end{array}$ & \\
\hline \multirow{2}{*}{ A1 } & 343 & 173 & $15-$ \\
& & & \\
A2 & 301 & 215 & $12-$ and \\
& 329 & 187 & $14-$ \\
A3 & 287 & 229 & $11-$ and \\
& 385 & 131 & $18-$ \\
A4 & 245 & 271 & $8-$ and \\
& 259 & 257 & $9-$ \\
A5 & 203 & 313 & $5-$ \\
\hline
\end{tabular}

Table II. Isomeric Distributions of Methyl Eicosapentaenoate Monohydroperoxides

\begin{tabular}{|c|c|c|c|c|c|c|c|}
\hline \multirow{2}{*}{$\begin{array}{l}\text { Reaction time } \\
(\mathrm{hr})\end{array}$} & \multirow{2}{*}{$\begin{array}{c}\text { POV } \\
(\mathrm{meq} / \mathrm{kg})\end{array}$} & \multicolumn{6}{|c|}{ Relative percentage $^{a}$} \\
\hline & & $5-\mathrm{OOH}$ & $\begin{array}{l}\text { 8- and } \\
9-\mathrm{OOH}\end{array}$ & $11-\mathrm{OOH}$ & $\begin{array}{l}12-\text { and } \\
14-\mathrm{OOH}\end{array}$ & $15-\mathrm{OOH}$ & $18-\mathrm{OOH}$ \\
\hline \multicolumn{8}{|c|}{ Myoglobin-catalyzed peroxidation } \\
\hline 2 & 1000 & 22 & 19 & 16 & 16 & 7 & 20 \\
\hline 4 & 1700 & 23 & 19 & 16 & 16 & 6 & 20 \\
\hline 8 & 3000 & 23 & 19 & 14 & 16 & 7 & 22 \\
\hline \multicolumn{8}{|l|}{ Autoxidation $^{b}$} \\
\hline 5 & 1200 & 22 & 19 & 14 & 16 & 7 & 22 \\
\hline
\end{tabular}

a Relative percentage was calculated from the peak areas of HPLC, and the relative percentage of 11- and 18isomers was calculated from the peak areas of GLC.

b Methyl eicosapentaenoate $(30 \mathrm{mg})$ in a test tube was allowed to stand for $5 \mathrm{hr}$ at $37^{\circ} \mathrm{C}$ under aerobic conditions.

$\beta-\gamma$ from the carbon-attached peroxy group can by cyclized to form endoperoxides. According to this, the 8-, 9-, 11-, 12-, 14- and 15-peroxy radicals of methyl eicosapentaenoate were able to cyclized to form the hydroperoxy endoperoxides, or to form the prostaglandin-like hydroperoxy bicyclic endoperoxides. Consequently, it is assumed that the predominance of outer isomers was based on the presence of these alternative pathways between the peroxy radicals.

The results of this study confirm that myoglobincatalyzed peroxidation of methyl eicosapentaenoate proceeds in a manner similar to the autoxidation of methyl eicosapentaenoate ${ }^{2,3)}$ and the non-enzymatic peroxidation of methyl arachidonate. ${ }^{7,8)}$

\section{REFERENCES}

1) A. L. Tappel, "Autoxidation and Antioxidants," Vol. 1, ed. by W. O. Lundberg, Wiley-Interscience, New York, 1961, pp. 325 366 .

2) R. Yamauchi, T. Yamada, K. Kato and Y. Ueno, Agric. Biol. Chem., 47, 2897 (1983).

3) R. Yamauchi, T. Yamada, K. Kato and Y. Ueno, Agric. Biol. Chem., 49, 2077 (1985).

4) E. N. Frankel, C. D. Evans, D. G. McConnel, E. Selke and J. Dutton, J. Org. Chem., 26, 4663 (1961).

5) E. N. Frankel, W. E. Neff, W. K. Rohwedder, B. P. S. Khambay, R. F. Garwood and B. C. L. Weedon, Lipids, 12, 1055 (1977). 
6) J. Terao and S. Matsushita, Agric. Biol. Chem., 45, 595 (1981).

7) S. Yamagata, H. Murakami, J. Terao and S. Matsushita, Agric. Biol. Chem., 47, 2791 (1983).
8) S. Yamagata, H. Murakami, J. Terao and S. Matsushita, Agric. Biol. Chem., 48, 101 (1984).

9) N. A. Porter, L. S. Lehman, B. A. Weber and K. J. Smith, J. Am. Chem. Soc., 103, 6447 (1981). 